http://rev.med.panacea.unica.edu.pe

Rev méd panacea. 2014; 4(3): 77-80.

Recibido: 30 de Noviembre del 2013 | Aceptado: 15 de Octubre del 2014 | Publicado: 31 de Diciembre del 2014

CONFICTOS DE INTERÉS: NO DECLARADOS

ORIGINAL BREVE

\title{
Utilidad del PPD para el descarte de la infección tuberculosa en estudiantes de medicina
}

\author{
María L. F. Acuña-Zevallos ${ }^{1, a}$, Ubaldo E. Miranda-Soberón ${ }^{1,2, b}$ \\ Facultad de Medicina, Universidad Nacional San Luis Gonzaga. Ica, Perú1 \\ Hospital Regional de Ica, Perú2 \\ Médico cirujanoa, medico especialista en pediatríab
}

\begin{abstract}
on el objetivo de determinar la utilidad del Tuberculin, Purified Protein Derivative (PPD) para el - descarte de la infección tuberculosa en estudiantes de medicina, se realizó un estudio transversal, en una muestra de 152 alumnos de la Facultad de Medicina de la Universidad Nacional San Luis Gonzaga que aceptaron participar en la investigación. La mayoría de los estudiantes presentaron PPD negativo (86,18\%), sin embargo 12 (7,89\%) presentaron resultados menores de $10 \mathrm{~mm}$ y nueve $(5,92 \%)$ presentaron resultados mayores de $10 \mathrm{~mm}$. El contacto con pacientes portadores de la enfermedad fue $1 \%$. Finalmente concluimos que a medida que el estudiante de medicina se expone a espacios de elevado riesgo para la transmisión de la tuberculosis, el porcentaje de resultados PPD positivos tiende a incrementarse. Por tanto, el uso del PPD como prueba de descarte de la infección tuberculosa es de utilidad.
\end{abstract}

Palabras clave: Tuberculosis; Prueba de Tuberculina; Estudiantes de Medicina (fuente: DeCS BIREME).

Autor corresponsal: María Luisa Fernanda Acuña Zevallos. Correo electrónico: maz.malu@yahoo.es

\section{INTRODUCCIÓN}

Desde hace muchos años la tuberculosis se ha convertido en un grave problema de salud pública(1,2). La Organización Mundial de la Salud (OMS) declaró en 1993 que la tuberculosis (TBC) era una emergencia mundial(3). Cerca de la tercera parte de la población mundial está infectada con el Mycobacterium tuberculosis y una de cada 10 contraerá tuberculosis activa en algún momento de su vida. En el 2008, se reportaron en el mundo más de 9,4 millones de casos nuevos y 1,8 millones fallecieron. El $95 \%$ de los casos y el $98 \%$ de las muertes por TBC ocurrieron en países en vías de desarrollo. Las muertes por TBC corresponden al $25 \%$ de la mortalidad evitable(4).

La TBC es una enfermedad infectocontagiosa prevenible y curable que se transmite por el aire(5). En la mayoría de los casos, el contagio directo se produce por vía pulmonar al inhalar aire con bacilos. También pueden ingerirse junto con alimentos contaminados (infección por vía digestiva)(6).

En la TBC del adulto, la clínica puede ser muy variada, desde cuadros agudos, hasta hallaz- gos radiológicos casuales en pacientes asintomáticos o con síntomas inespecíficos (astenia, anorexia, pérdida de peso, febrícula, sudoración nocturna). El eritema nodoso es frecuente al inicio de la infección tuberculosa, así como los cuadros bronconeumónicos, siendo ya menos habitual la hemoptisis. Para su diagnóstico existen cuatro pruebas disponibles: el PPD, el estudio radiológico de tórax, la baciloscopía y el cultivo(7).

El personal de salud no es ajeno a esta enfermedad, el antecedente más serio ocurrió en 1997, cuando se produjo un brote de tuberculosis en el Hospital Nacional Guillermo Almenara de EsSalud. La tasa previa en 1994 fue de 167 casos x 100000 habitantes, en el brote de 1997 fue de $6977 \times 100000$ habitantes en el servicio de laboratorio y $932 \times$ 100000 habitantes en los servicios de medicina. Se confirmó TB activa en 36 trabajadores y el único factor de riesgo para los de laboratorio, fue el uso de áreas comunes(5).

En general, para infectarse de TB se requiere un contacto intenso y prolongado con una persona bacilífera. En el contagio influyen factores como la cantidad de bacilos en la expectoración del enfermo, el grado de intimidad, la duración de la exposición, la edad del contacto(8). Así, dentro del personal de mayor riesgo en la transmisión de tuberculosis nosocomial se encuentran los estudiantes de medicina(9). En un estudio realizado por Madrigal y Vazquez (10), se halló que al comparar estudiantes de carreras biomédicas (medicina, enfermería y estomatología), con estudiantes de carreras no biomédicas (contaduría pública) de una Universidad de México, los estudiantes de biomédicas en contacto con pacientes en su práctica clínica tienen riesgo de contagio con una respuesta adecuada, sin desarrollar enfermedad. En el 2000, en el Hospital Nacional Daniel A. Carrión, de 98 internos y médicos residentes evaluados por PPD, 59\% fueron positivos al momento de la evaluación, 36 inicialmente negativos, 6 convirtieron a positivos y 2 adquirieron TB activa (5).

El despistaje continuo y quimioprofilaxis según necesidades en pacientes de diversos servicios, personal, estudiantes y otros usuarios de los servicios de salud contempla la vigilancia de viraje de PPD (11). Por ello, se plantea la presente investigación con el objetivo de determinar la utilidad del PPD para el descarte de la infección tuberculosa en los estudiantes de Medicina Humana en el periodo 2010 - 2011.

\section{MATERIALES Y MÉTODOS}

Se realizó un estudio transversal, con el objetivo de conocer la utilidad del PPD para el descarte de la infección tuberculosa en los estudiantes de Medicina Humana en el periodo 2010 - 2011. La muestra estuvo constituida por 152 alumnos de la Facultad 
de Medicina Humana "Daniel Alcides Carrión te las visitas médicas y uno (0,7\%) de ellos de García" de la Universidad Nacional San Luis forma extrahospitalaria con paciente TB coGonzaga de Ica que aceptaron participar en la nocido. investigación.

Asimismo, se elaboró un formulario de recolección de datos, donde se analizan las variables en estudio y se aplicó la prueba de tuberculina -técnica habitual para diagnosticar la infección tuberculosa- (PPD).

El PPD es un derivado proteico purificado obtenido tras el filtrado de cultivo de Mycobacterium tuberculosis esterilizado y concentrado. La técnica consiste en la inyección intradérmica con una aguja del calibre 27 en la cara anterior del antebrazo, $(0,1 \mathrm{ml})$ de 2 unidades tuberculina PPD RT-23, en una zona donde no existan lesiones cutáneas. Debe producirse una pápula de 6-10 $\mathrm{mm}$ de diámetro para que la técnica sea correcta. Su lectura se realiza a las 72 horas de la inyección, midiendo el diámetro transversal de la induración según el eje longitudinal del antebrazo, y su resultado se mide en milímetros $(\mathrm{mm})$. En el caso de no existir induración sino únicamente eritema, se interpreta como o mm, sí el diámetro de la pápula es mayor de $10 \mathrm{~mm}$ se considera positivo o sospechoso.

Los datos obtenidos de nuestro estudio se ordenaron, analizaron y procesaron a través del programa estadístico SPSS versión 18.0. Asimismo el análisis de las variables se realizó calculando las frecuencias y proporciones de las variables cualitativas.

\section{RESULTADOS}

De los estudiantes evaluados, más del 80,93\% estaba entre el primer y sexto ciclo. 12 $(7,98 \%)$ de ellos estaban en el cuarto año académico, $13(8,55 \%)$ en el quinto año y 4 $(2,63 \%)$ en el sexto año.

La edad promedio de los estudiantes fue $21,25 \pm 0,25$ años: De ellos 79 fueron de sexo masculino con edad promedio $21,38 \pm 0,3$ años y 73 de sexo femenino cuya edad promedio fue 21,11 $\pm 0,4$ años.

El $73 \%$ de los estudiantes eran nativos de la ciudad de Ica, 9,2\% provenían del departamento de Ayacucho, 9,9\% del departamento de Lima y 7,9\% de otros departamentos.

En ellos, 14 (9,2\%) habían presentado tos mayor de tres semanas en el último año y 18 $(11,18 \%)$ presentaban tos al momento del estudio.

En relación a los contactos, tres $(2,0 \%)$ de los estudiantes habían tenido contacto con un tosedor crónico que recibió tratamiento para TB y seis (3,9\%) con un tosedor crónico en el lugar donde vivía. Dos (1,3\%) estudiantes habían tenido contacto con pacientes TB duran-
En los resultados de PPD se halló que en el mayor porcentaje de los estudiantes (131; $86,18 \%$ ) no hubo cambios; mientras que en 12 $(7,89 \%)$ hubo cambios menor o igual a $10 \mathrm{~mm}$. En $9(5,92 \%)$ de los estudiantes evaluados se halló resultado mayor de $10 \mathrm{~mm}$.

Este resultado fue similar en relación al año académico que cursaban. Sin embargo, en aquellos estudiantes que estaban entre el cuarto y sexto año académico el porcentaje de resultado positivo para PPD fue mayor $(13,8 \%)$ que en aquellos que estaban en años académicos inferiores (4,1\%).

\section{DISCUSIÓN}

La tuberculosis es una enfermedad ocupacional importante entre los trabajadores sanitarios y la reducción de su riesgo debería ser una prioridad(10,12). Los estudiantes de ciencias de la salud, entre ellos los que cursan la carrera de medicina, son individuos que podrían tener tuberculosis con o sin diagnóstico establecido $y$, por lo tanto, potencialmente contagiosos (bacilíferos), lo que los expone al contagio con la bacteria como parte de su formación profesional. Así, Accinelli y col(13), halló que un tercio de los pacientes profesionales de salud atendidos en el programa de control de la tuberculosis eran estudiantes de ciencias de la salud y la mayoría estudiantes de medicina.

En los resultados de nuestra muestra se observa que la frecuencia del PPD positivo es $6 \%$, resultado posiblemente afectado por la distribución de alumnos según año de estudio. La concentración de estudiantes de los primeros años es mayor que en los últimos años. Estudios realizados $(3,10,12,13,14,15)$, señalan que la prevalencia de tuberculosis es baja entre los estudiantes de primer año que ingresan a la profesión médica pero aumenta con cada año de entrenamiento, donde para mejorar sus destrezas tienen que estar más tiempo en contacto con los enfermos y sus familiares, mientras que los internos de medicina, por las condiciones de su estancia, pasan aún más tiempo en contacto con los pacientes y muchas veces sin tener la experiencia adecuada tienen que afrontar situaciones de alto riesgo como el contagio de enfermedades infecto contagiosas (16).

En nuestra muestra, no se hizo evaluación previa de PPD. Sin embargo, se observó que los estudiantes que cursaban los primeros años de estudio y aún no realizaban prácticas clínicas dentro del currículo de estudios el resultado positivo para PPD fue $4,1 \%$, mientras que los estudiantes de los años en que se realizaban prácticas clínicas hospitalarias, el resultado PPD positivo fue mayor (13,8\%), a pesar que este grupo tuvo baja representación en la muestra estudiada y no se incluyó a estudiantes del internado hospitalario. Bonifacio(17), aunque en una población diferente (internos y residentes de medicina) pero con mayor exposición al riesgo de contagio por tuberculosis, halló elevado porcentaje de resultados PPD positivos que alcanzó un 17\%. Madrigal y col(10), además, halló que las estudiantes de enfermería muestran un incremento evidente en la respuesta al PPD en los últimos semestres, posiblemente debido a que se encuentran en contacto más cercano con los pacientes por el perfil profesional que les compete. Fernández(18), halló que los estudiantes de las otras facultades de ciencias de la salud inician sus estudios universitarios con niveles más altos de infección tuberculosa que los de medicina y al terminar la carrera, que es más corta, no alcanzan los niveles de infección que los estudiantes de medicina. Como vemos, en diferentes estudios, más años de formación clínica y una mayor exposición a los enfermos de tuberculosis son factores de riesgo para una infección nueva lo que provee un soporte adicional para la transmisión nosocomial.

No obstante, no se debe dejar de lado los resultados con viraje menor de $10 \mathrm{~mm}$ que en nuestra muestra representan el $8 \%$. Si bien puede deber a la exposición a la vacuna BCG, el viraje entre 5 y $10 \mathrm{~mm}$ puede calificarse como sospechoso(10).

Es importante mencionar que en el estudio realizado por Arbaiza(3), el examen bacteriológico de esputo con la tinción de ZiehlNeelsen y la investigación radiológica fotoroentgen (Rayos X) no mostraron resultados de tuberculosis activa en una población similar; sin embargo, se hallaron resultados positivos al examen inmunológico, mediante la intradermorreacción, Test PPD.

Finalmente concluimos que a medida que el estudiante de medicina se expone a espacios de elevado riesgo para la transmisión de la tuberculosis, el porcentaje de resultados PPD positivos tiende a incrementarse. Por tanto, el uso del PPD como prueba de descarte, es de utilidad para la detección oportuna de la infección tuberculosa.

Asimismo recomendamos la implementación de un programa de control de TB en las facultades de medicina, lo que permitiría el diagnóstico oportuno y la administración oportuna de tratamiento preventivo en los casos que se consideren necesarios. 


\section{REFERENCIAS BIBLIOGRÁFICAS}

1. Organización Mundial de la Salud. Programas y Proyectos. Nota descriptiva Nº4 Mayo de 2010. [Link]

2. Valdés G, Fernández A. Situación actual de la tuberculosis y su manejo terapéutico. Revista 16 de Abril. Revista Científico Estudiantil de las Ciencias Médicas de Cuba. Año 2010. [Link]

3. Arbaiza J. Estudio inmunológico, clínico bacteriológico, radiológico de tuberculosis en estudiantes de medicina. Cultura rev. asoc. doc. USMP. 2002; 20(16):13-27.

4. Silva V, Cunha A, Kritski A. Prueba cutánea de tuberculina entre estudiantes de medicina de un Hospital Docente de Río de Janeiro. Infection control and hospital Epidemiology. 2002; 23(10): 591-594. [Link]

5. Ministerio de Salud del Perú. Control de infección tuberculosa en trabajadores de salud. Año 2007. [Link]

6. Túñez B, García R, Pérez $\mathbf{M}$ y Lado. Epidemiología de la tuberculosis. Med Integral. 2002; 39(5):172-180.

7. Martincano J. Tuberculosis Respiratoria. Documentos clínicos SEMERGEN. 1992; 1 (1): 33-49.

8. Ruiz J, Blanquer R, Calpe J et al. Diagnóstico y Tratamiento de la Tuberculosis. Arch Bronconeumol. 2008; 44(10):551-566.

9. Rodriguez M. Tuberculosis. [Link]

10. Madrigal A. Reactividad al PPD en estudiantes de ciencias biomédicas de la Universidad Autónoma de Aguascalientes, México 2005. Revista de Enfermedades Infecciosas en Pediatría 2008; 21(83) 68-74.

11. Murray, Rosenthal y Pfaller. Microbiología Médica. 6ta Edición. 2009.

12. Emadi-Koochak H. Tuberculin Skin Test Conversion among Students during their Educational Course in Medical and Pharmacy Schools: A Multiple Cohort Study. NRITLD (National Research Institute of Tuberculosis and Lung Disease) Iran. Tanaffo. 2009; 8(4), 33-36

13. Accinelli R. Enfermedad tuberculosa entre trabajadores de salud. Acta Med Per. 2009; 26(1) 35-47.

14. Accinelli R, Alvarez L, Valles P. Annual risk of tuberculosis infection among medical students of Universidad Peruana Cayetano Heredia. American Journal of Respiratory and Critical Care Medicine. 2002; 165 (8):439.

15. Joshi R, Reingold AL, Menzies D, Pai M. Tuberculosis among health-care workers in low- and middle-income countries: a systematic review. PLoS Med 2006; 3 (12):494.

16. Alarco JJ. Importancia de la prueba de Tuberculina en estudiantes de medicina. Rev méd panacea. 2012; 2(3): 102-3. [Link]
17. Bonifacio N, M Saito, RH Gilman, F Leung, NC Chávez, JC Huarcaya, et al. Alto riesgo de tuberculosis en el Hospital de Médicos, Perú. Emerg Infect Dis 2002; 8(1):747-748.

18. Fernández E. Riesgo de adquirir infección tuberculosa en estudiantes de medicina. [Tesis para optar el Título de Médico Cirujano]. Universidad Peruana Cayetano Heredia. 1987. $39 \mathrm{pp}$ 
FINANCIAMIENTO: Autofinanciado.

AGRADECIMIENTOS: A los estudiantes de la facultad de medicina de la Universidad Nacional San Luis Gonzaga, por su participación activa para la realización de este estudio.

CONTRIBUCIONES DE AUTORÍA: MLFAZ y UEMS participaron en el diseño del estudio, el análisis de los datos, revision crítica del artículo y aprobación de la versión final.

\section{PPD utility for disposal of infection TB among medical students}

I $\mathrm{n}$ order to determine the usefulness of Tuberculin, Purified Protein Derivative (PPD) for the disposal of tuberculosis infection among medical students, a cross-sectional study was conducted on a sample of 152 students of the Faculty of Medicine of the National University San Luis Gonzaga who agreed to participate in the investigation. Most students had negative PPD (86.18\%), however 12 (7.89\%) presented results under $10 \mathrm{~mm}$ and nine $(5.92 \%)$ had greater results than $10 \mathrm{~mm}$. Contact with patients with the disease was $1 \%$. Finally we conclude that as the medical student is exposed to high-risk areas for TB transmission, the percentage of positive PPD results tends to increase. Therefore, the use of PPD as evidence of discarding of tuberculosis infection is useful.

Key words: Tuberculosis; Tuberculin Test; Medical Students . (source: MeSH NLM)

\section{CITA SUGERIDA.}

Acuña-Zevallos MLF , Miranda-Soberón UE. Utilidad del PPD para el descarte de la infección tuberculosa en estudiantes de medicina. Rev méd panacea.2014; 4(3):77-80. 